\title{
Performance of Adaptive Modulation Techniques in the UMTS System*
}

\author{
Francesco Alesiani Ezio Biglieri Giorgio Taricco Emanuele Viterbo \\ Politecnico di Torino - Dipartimento di Elettronica
}

\begin{abstract}
We study the performance of a UMTS downlink, as achieved by a single-user (RAKE) and a multiuser (linear MMSE) receiver with adaptive modulation and multicode in the presence of multipath fading. We advocate an adaptive transmission scheme that varies the number of virtual users and the modulation spectral efficiency in order to optimize the system throughput. Two multipath channel models are considered, exhibiting 3 and 9 paths, respectively. Over the more favorable multipath channel, the multiuser MMSE receiver yields a significant performance enhancement with respect to the RAKE receiver.
\end{abstract}

\section{INTRODUCTION}

To enable future-generation mobile systems to deliver flexible services, modulation schemes with high spectral efficiencies are called for. Since wireless channels exhibit fading, viz., a time-varying response, adaptive transmission strategies look attractive to achieve higher channel capacity.

The basic idea behind adaptive transmission consists of allocating power and rate to take advantage of favorable channel conditions by transmitting at high speeds, while at the same time counteracting bad conditions by reducing the throughput. For an assigned quality of service (QoS), the goal is to increase the average spectral efficiency by using the knowledge of the channel state at the transmitter. In principle, the achievable performance improvement can be evaluated by computing the Shannon capacity of a given channel with and without it. However, it should be kept in mind that capacity results refer to a situation in which complexity and delay are not constrained. The results of [10] show that for an iid Rayleigh fading channel the capacity with channel state information (CSI) at the transmitter and the receiver is only marginally larger than without it. However, in a delay- and complexity-constrained environment a considerable gain can be achieved (see, e.g., [6]).

Adaptive techniques are based on the measurement of the parameters describing the quality of the transmission channel and on the selection of transmission parameters based on the optimization of a preassigned cost function.

A basic assumption here is that the channel does not vary too rapidly. Thus, adaptive techniques can only be beneficial in a situation where the Doppler spread is relatively low

\footnotetext{
* This research was supported by Omnitel Vodafone, Italy.
}

such as an indoor environment. In these conditions, adaptive techniques can work on a frame-by-frame basis.

The simplest propagation parameter that can be used to assess the quality of the transmission channel is the received signal level which is proportional to the SNR in the absence of interference. In all instances, accurate channel estimation and reliable feedback path between $T X$ and $R X$ are required and can be accomplished by using either a dedicated channel or a suitable duplex system. The simplest duplex arrangement is probably time-division duplex (TDD), where both TX and RX transmit over the same channel, and hence experience similar fading conditions as their transmissions are typically half a TDD frame apart. Frequency-division duplex (FDD) can also be used if the carrier spacing is not too large with respect to the coherence bandwidth of the fading.

The accuracy of the channel estimate influences the performance of the adaptive system. With perfect channel estimation at the transmitter, a frequency-nonselective Rayleigh fading channel is transformed into an AWGN channel with a modified SNR. However, the effective channel conditioned on outdated fading estimates has a Rician distribution, and may become nearly Rayleigh distributed when fading is fast [9]. Thus, the issue of robustness of the adaptive scheme to inaccuracies of channel estimates needs investigation.

In this paper these concepts are applied to UMTS, the 3rdgeneration mobile system, where adaptive modulation techniques (AMT) are advocated with the goal of achieving a downstream bit rate of $2 \mathrm{Mbit} / \mathrm{s}$.

\section{A TAXONOMY OF ADAPTIVE TRANSMISSION TECHNIQUES}

In a nutshell, adaptive transmission transforms the random SNR of the channel into a constant one, which can be obtained by varying adaptively one or more of the following parameters:

1. Transmitted power level

2. Symbol transmission rate

3. Constellation size

4. Code rate

Adapting power level. Through power control, only the transmission level is varied according to the channel fluctuations (see, e.g., [6]). This strategy increases the TX peakpower requirements, and, in a multiuser environment, the 
level of cochannel interference, which may reduce channel capacity if coordination among users is not implemented.

Adapting constellation size. It consists of transmitting at the highest possible rate compatible with an assigned quality of service (QoS) by using a hierarchy of different constellations of increasing size. Among adaptive transmission techniques, adaptive modulation plays a central role, because it increases the data transmission efficiency without increasing the multiaccess interference power.

Adaptive constellation size may be implemented so as to maintain a constant transmit power while providing a target QoS (see $[13,22])$. The number of signals in the modulator constellation can be varied in such a way that the short-term BER is approximately constant while the short-term bit rate varies, or vice versa.

In [11] it is shown that a single-user environment adaptive modulation can provide a $5-10-\mathrm{dB}$ gain over a fixed-rate system having only power control.

Adapting code rate. The coding scheme can be changed so as to respond to the channel state by selecting the optimum code rate (see, e.g., [21]). Punctured convolutional codes [12, 24] are especially useful to this purpose, because they enable adaptive encoding and decoding without modifying the basic structure of the encoder and the decoder; however, other coding schemes have been advocated as well [23].

Adapting power level and constellation size. In ref. [11] both modulation scheme and transmit power are adapted in a single-user environment. In [18], joint power control and adaptive modulation is considered for a multiuser channel. It is shown that this combination leads to a significant throughput increase as compared to no power control.

Adapting constellation size and symbol rate. In [19], both constellation size and symbol transmission rate are adapted. The system selects the optimum modulation parameters so as to maximize the bit rate while satisfying the required BER. Here a lower symbol rate is achieved by consecutively transmitting identical QAM symbols ar the maximum symbol rate $R_{\max }$ (this is equivalent to repetition coding). With this solution, both TX and RX need only one lowpass filter with bandwidth $R_{\max }$.

Adapting power and transmission rate. The approach taken in [11] is to optimize both the transmission rate and the power so as to maximize the spectral efficiency while satisfying average power and BER constraints. Refs. [14, 15] advocate an adaptive-spreading-factor, adaptive-power system. In [20] the adaptation is formulated for CDMA uplinks as a throughput-optimization problem in terms of the spreading gains (equivalently, transmission bit rates) and the transmit powers of the users.

Adapting modulation size and coding scheme. This adaptation strategy refers to trellis- (or, more generally, coset-) coded modulation. Here the number of subsets is kept constant, while the number of signals per subset is adapted. By holding the number of information bits entering the encoder fixed, and adapting the number of uncoded bits according to the estimate of the channel state, the trellis structure is changed so as to vary the number of parallel transitions. As observed in [17], this adaptation scheme is not robust to estimation errors: in fact the presence of parallel transitions (which have diversity 1) impairs performance in environments close to the Rayleigh fading channels. If this is the case, bit-interleaved coded modulation (BICM) [5], which consists of separating encoder and modulator with a bit interleaver, may be a more attractive proposition, because it increases the time diversity. With this scheme, the code is left fixed, while the signal constellation is adapted to the channel conditions (hence, it should be categorized under the rubric "adapting the constellation size"). The analysis in [17] suggests that the code structure of BICM is more suitable for adaptive systems that must support highly mobile users.

\section{ADAPTIVE MODULATION}

In order to match the transmission rate to the channel conditions, different transmission modes are considered. For a fixed spreading factor $L$, the transmission modes are defined by the number of virtual users $\left(N_{\mathrm{vu}}=1, \ldots, L-1\right)$ and the modulation spectral efficiency $(\eta=2,4,6 \mathrm{bit} / \mathrm{s} / \mathrm{Hz})$. The number of virtual users corresponds to the number of spreading codes used in the multicode transmission, while the different spectral efficiencies are obtained by using QPSK, 16QAM and 64-QAM modulation schemes. Note that one spreading code is always reserved for the common pilot channel. Hence, its overhead may be reduced by increasing $L$. Since UMTS employs orthogonal Hadamard spreading codes, the maximum number of virtual users is $L-1$.

As an example we list in Table 1 the achievable gross information bit rates using a rate- $1 / 3$ channel code for $L=4$ in the different modes. The number of data bits per spreading code with $L=4$ is 1248 when QPSK is used. Furthermore, 16 bits are used to generate pilot symbols, 8 bits for the Transport Format Combination Indicator (TFCI) and 8 bits for the Transmit Power Control (TPC).

\begin{tabular}{|c|c|c|c|}
\hline \multirow{2}{*}{$N_{\mathrm{vu}}$} & \multicolumn{3}{|c|}{$\eta$} \\
\cline { 2 - 4 } & $2 \mathrm{bit} / \mathrm{s} / \mathrm{Hz}$ & $4 \mathrm{bit} / \mathrm{s} / \mathrm{Hz}$ & $6 \mathrm{bit} / \mathrm{s} / \mathrm{Hz}$ \\
\hline \hline 1 & $624 \mathrm{kbps}$ & $1248 \mathrm{kbps}$ & $1872 \mathrm{kbps}$ \\
2 & $1248 \mathrm{kbps}$ & $2496 \mathrm{kbps}$ & $3744 \mathrm{kbps}$ \\
3 & $1872 \mathrm{kbps}$ & $3744 \mathrm{kbps}$ & $5616 \mathrm{kbps}$ \\
\hline
\end{tabular}

Table 1: Information bit rates for the different transmission modes

Switching between transmission modes represented by the pairs $\left(N_{\mathrm{vu}}, \eta\right)$ is dictated by the value of the SINR estimated from the pilot channel in the previous slot.

We hasten to observe that additional redundant information must be carried by each frame in order to identify which 
modulation scheme was used. Hereafter we do not take this into account, and assume that the scheme used is accurately identified by the receiver.

\section{A. Turbo encoding and decoding}

Our simulations were based on the rate- $1 / 3$ Turbo code specifications given in document [1]. For all transmission modes we assumed that the code words contain the data transmitted by all virtual users during one slot, whose duration is $0.667 \mathrm{~ms}$. Thus, their lengths change according to the transmission mode.

In order to combine turbo decoding with higher-order modulations, an additional block called the bit-metric computer evaluates the log-likelihood ratios of each bit labeling the modulated signal. The decoder uses iterative decoding up to 8 iterations. In order to evaluate and compare the error rates for the different transmission modes in an adaptive setting, we assume that each code word (slot) carries $N_{\mathrm{vu}}=3 \times \eta / 2$ data packets of finite length. For example, with $L=4$ the transmission mode $\left(N_{\mathrm{vu}}=3,16-\mathrm{QAM}\right)$ allows transmission of six data packets. Note that data packets are virtual units of information bits. For this reason we consider as performance parameters the packet error rate (PER) and the system throughput defined as $R_{b} \times(1-\mathrm{PER})$. Here $R_{b}$ is the gross information bit rate (which includes packet errors), as given for example in Table 1.

The modulator is implemented according to document [2, sec. 5] with the additional feature of producing multilevel modulated symbols from 16- and 64-QAM constellations. We note that pilot symbols are always transmitted with QPSK for all transmission modes. The Common Pilot Channel transmits the complex symbol $(1+) j$ with the same power of the average transmitted power used for the data symbols. For all modulation schemes Gray bit labeling was selected [5]. Spreading is implemented, according to document [2, sec. 5], using Orthogonal Variable Spreading Factor (OVSF) codes.

\section{B. Fast power control}

The transmitted signal is power-controlled with 1-dB steps at the slot frequency. The channel level is measured in each slot and a transmit-power UP or DOWN is performed accordingly for the following slot (see [3]).

\section{Fading channel}

The multipath fading channel model was selected from those given in Annex $\mathrm{C}$ of [4]. All the results given in this paper were based on two power-delay profiles conventionally denoted as "Pedestrian A" (good) and "Pedestrian B" (bad).

The time-varying nature of the channel, caused by the relative motion of the receiver with respect to the base station, is modeled according to the standard Jakes' model. In all the following it is assumed that the mobile speed is $3 \mathrm{~km} / \mathrm{h}$, corresponding to a Doppler band of $5 \mathrm{~Hz}$. In our simulations, the Jakes' Doppler spectrum was approximated by using a three-pole discrete filter.

\section{Receiver}

We consider a RAKE receiver with a number of fingers equal to the number of paths in the multipath channel. Perfect CSI is assumed. The receiver performs maximum-ratio combining of all paths. The output of the receiver is passed to the despreading filters, which act as single-user matched filter detectors for all virtual users. Due to the presence of multipath fading, the received signature waveforms are nonorthogonal: hence, this solution is sub-optimal and its performance depends on $N_{\mathrm{vu}}$. Additionally, we consider a linear multiuser MMSE receiver whose discrete-time baseband model is derived following [16].

\section{ADAPTIVE SELECTION OF TRANSMISSION MODES}

Let $E$ denote the random variable defining the occurrence of a decoding error: $E=0$ for correct packets an 1 for packet errors. Let $\gamma$ denote the SINR, and $p(\gamma, E=) e$ be the joint probability density function (pdf) of the SINR and the decoding outcome $E(e=0,1)$. These pdf's depend on the transmission mode and on the value of $E_{b} / N_{0}$. They can be estimated for the given channel during a training period. We select $M$ transmission modes and denote each of them by an index $\mu=1, \ldots, M$. Transmission mode $\mu$ corresponds to the pdf $p_{\mu}(\gamma, E=) e$ and to the bit rate $R_{b, \mu}$. Since our goal is to achieve reliable transmission at the maximum bit rate, a maximum a-posteriori (MAP) adaptive receiver can be operated by selecting the mode

$$
\widehat{\mu}(\gamma)=\arg \max _{\mu: p_{\mu}(\gamma, E=0)>p_{\mu}(\gamma, E=1)} R_{b, \mu}
$$

This rule translates into a set of thresholds delimiting the intervals where $\widehat{\mu}(\gamma)$ takes on a fixed value. As an example, we consider the UMTS RAKE receiver with spreading factor $L=4$ and the following transmission modes:

$$
\begin{aligned}
& \text { 1. } N_{\mathrm{vu}}=2, \text { QPSK } \\
& \text { 2. } N_{\mathrm{vu}}=3, \text { QPSK } \\
& \text { 3. } N_{\mathrm{vu}}=3,16-\mathrm{Q} A M \\
& \text { 4. } N_{\mathrm{vu}}=3,64-\mathrm{QAM}
\end{aligned}
$$

In this case, the thresholds obtained are approximately $-5,1$, and $4 \mathrm{~dB}$ for values of $E_{b} / N_{0}$ ranging from 6 to $10 \mathrm{~dB}$. For different values of $E_{b} / N_{0}$, a slight change is expected but, in our simulations, we kept these values fixed.

\section{Simulation Results}

In this section we report simulation results relevant to the downlink of a UMTS CDMA system with a rate-1/3 turbocode defined according to [1], spreading factor $L=4$, chip rate of $3.84 \mathrm{Mchip} / \mathrm{s}$, slot duration of $10 \mathrm{~ms}$ (each slot carrying one codeword), fast power control in 1-dB steps, and 
Walsh-Hadamard spreading codes. We consider a multipath frequency-selective Rayleigh fading channel described by the Pedestrian A/B models [4].

Figures 1 and 2 report the results obtained by using a single-user RAKE receiver and a multiuser MMSE receiver, respectively. Each figure contains four plots: those at left refer to the Pedestrian A channel model; those at right refer to the Pedestrian B model; the upper ones show the throughput and the lower ones report the PER. Simulation runs are 10 seconds long (i.e., 1000 slots). Power control increases the average value of $E_{b} / N_{0}$ by $4.5 \mathrm{~dB}$.

Each diagram contains results referring to nine fixed transmission modes $\left(N_{\mathrm{vu}}, \eta\right)$ for $N_{\mathrm{vu}}=1,2,3$ and $\eta=1,2,3$ and the adaptive transmission scheme using the transmission modes $\left(N_{\mathrm{vu}}, \eta\right)=(2,2),(3,2),(3,4)$, and $(3,6)$.

The results show an overall performance enhancement of the multiuser MMSE receiver that is particularly noticeable with the Pedestrian A channel model. With the Pedestrian B model the improvement is limited since the most demanding modes are not achievable. However, we note a considerable reduction of the PER with the MMSE receiver.

It can be seen that the performance of the adaptive receiver is better than the performance of any fixed mode receiver over the whole range of $E_{b} / N_{0}$. Additionally, the adaptive receiver performance degrades smoothly while the $E_{b} / N_{0}$ is reduced in contrast with the abrupt degradation characteristic of fixed-mode receivers.

\section{REFERENCES}

[1] "3GPP Technical specification Group Radio Access Network; Multiplexing and channel coding (FDD)," 1999, [3G TS 25.212].

[2] "3GPP Technical specification Group Radio Access Network; Spreading and modulation (FDD)," 1999, [3G TS 25.213].

[3] “3GPP Technical specification Group Radio Access Network; Physical layer procedures (FDD)," 1999, [3G TS 25.214].

[4] “Annex C of UTRA RTT proposal — RTT Revision - Performance Results," Sep. 1998, [Tdoc SMG2 351/98].

[5] G. Caire, G. Taricco, and E. Biglieri, "Bit-interleaved coded modulation,” em IEEE Trans. Inform. Theory, Vol. 44, No. 3, pp. 927-946, May 1998.

[6] G. Caire, G. Taricco, and E. Biglieri, "Optimum power control over fading channels," IEEE Trans. Inform. Theory, Vol. 45, No. 5, pp. 1468-1489, July 1999.

[7] P. Chow, J. M. Cioffi, and J. A. C. Bingham, "A practical discrete multitone transceiver loading algorithm for data transmission over spectrally shaped channels," IEEE Trans. Commun., pp. 773-775, Feb.-March 1995.

[8] S. Diggavi, "Analysis of multicarrier transmission in timevarying channels," Proc. IEEE Int. Conf. Commun., Montreal, Canada, pp. 1191-1195, June 8-12, 1996.

[9] D. Goeckel, "Adaptive coding for time-varying channels using outdated fading estimates," IEEE Trans. Commun., Vol. 47, No. 6, pp. 844-855, June 1999.
[10] A. J. Goldsmith, "Capacity and dynamic resource allocation in broadcast fading channels," Proc. Allerton Conf. Communications, Control, and Computing, Monticello, IL, pp. 915-924, 1995.

[11] A. J. Goldsmith and S.-G. Chua, "Variable-rate variablepower MQAM for fading channels," IEEE Trans. Commun., Vol. 45, No. 10, pp. 1218-1230, October 1997.

[12] J. Hagenauer, "Rate-compatible Punctured Convolutional Codes (RCPC codes) and their applications," IEEE Trans. Commun., Vol. 36, No. 4, pp. 389-400, Apr. 1988.

[13] L. Hanzo, "Bandwidth-efficient wireless multimedia communications," Proceedings of the IEEE, Vol. 86, No. 7, pp. 13421382, July 1998.

[14] C.-L. I and K. K. Sabnani, "Variable spreading gain CDMA with adaptive control for true packet switching wireless networks," Proc. of ICC'95, pp. 725-730, Seattle, WA, June 1822, 1995.

[15] C.-L. I and K. K. Sabnani, "Variable spreading gain CDMA with adaptive control for integrated traffic in wireless networks," Proc. of VTC'95, pp. 794-798, July 25-28, 1995.

[16] A. Klein, G.K. Kaleh, and P.W. Baier, "Zero forcing and Minimum Mean-Square-Error Equalization for Multiuser Detection in Code-Division Multiple-Access Channels," IEEE Transactions on Vehicular Technology, Vol. 45, No. 2, pp. 276-287, May 1996.

[17] P. Örmeci, X. Liu, D. L. Goeckel, and R. D. Wesel, "Adaptive bit-interleaved coded modulation," submitted for publication, 1999.

[18] X. Qiu and K. Chawla, "On the performance of adaptive modulation in cellular systems," IEEE Trans. Commun., Vol. 47, No. 6, pp. 884-895, June 1999.

[19] T. Ue, S. Sampei, and N. Morinaga, "Symbol rate and modulation level controlled adaptive modulation systems with TDMA/TDD for high bit rate transmission in high delay spread environments," Electronics Letters, Vol. 32, No. 4, pp. 304-305, 15th Feb. 1996.

[20] S. Ulukus and L. J. Greenstein, "Throughput maximization in CDMA uplinks using adaptive spreading and power control," ISSSTA 2000, Parsippany, NJ, Sept. 6-8, 2000.

[21] B. Vucetic, "An adaptive coding scheme for time-varying channels," IEEE Trans. Commun., Vol. 39, No. 5, pp. 653663, May 1991.

[22] W. T. Webb and R. Steele, "Variable rate QAM for mobile radio," IEEE Trans. Commun., Vol. 43, No. 7, pp. 2223-2230, July 1995.

[23] K. Wu, S. Lin, and M. Miller, "A hybrid ARQ scheme using multiple shortened cyclic codes," Proc. IEEE GLOBECOM, Miami, FL, pp. C8.61-C8.65, 1982.

[24] Y. Yasuda, K. Kashiki, and Y. Hirata, "High rate punctured convolutional codes for soft decision Viterbi decoding," IEEE Trans. Commun., Vol. 32, No. 3, pp. 315-319, Mar. 1984. 

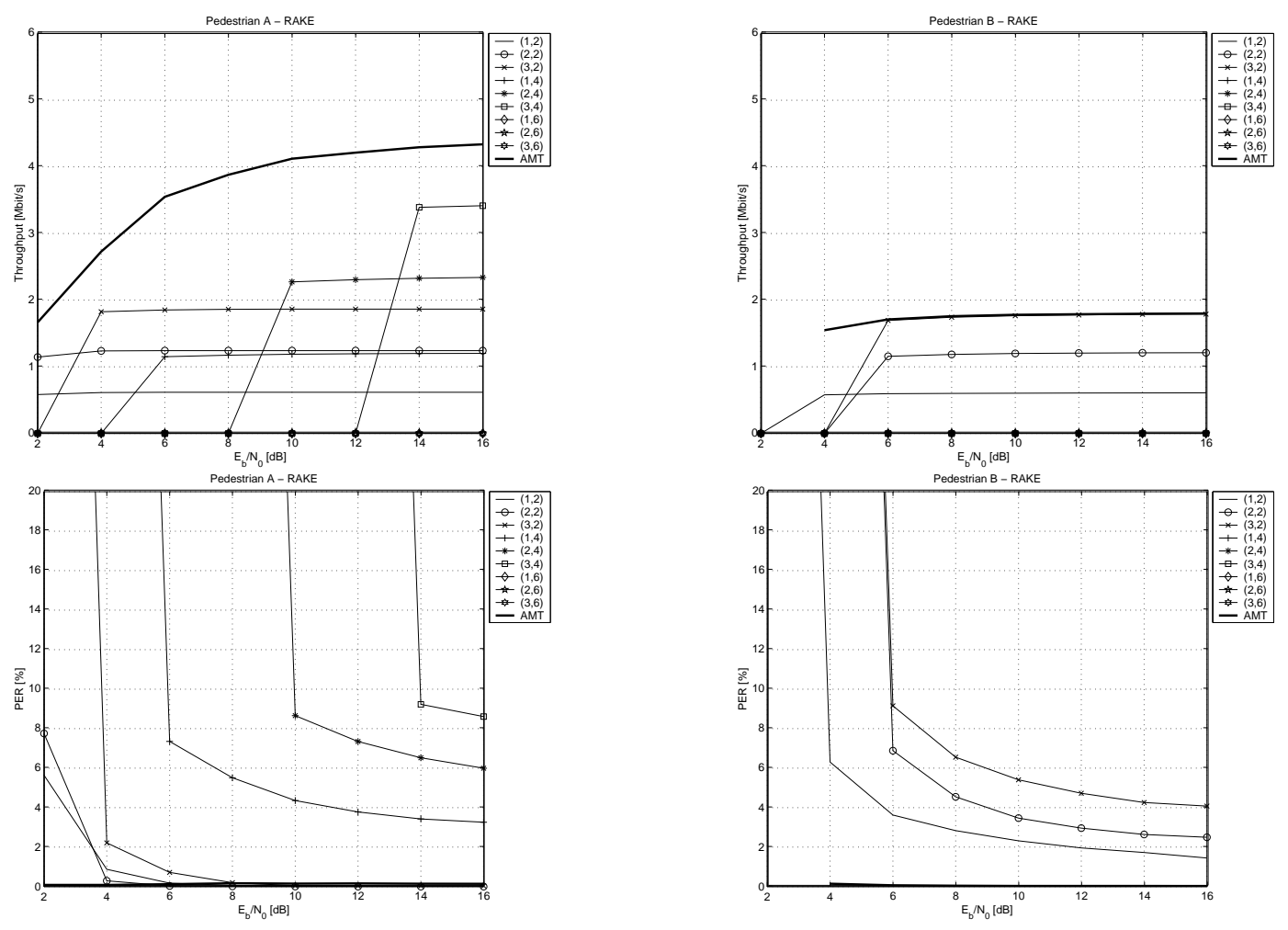

Figure 1: Simulation results (throughput and PER) for the single-user RAKE receiver.
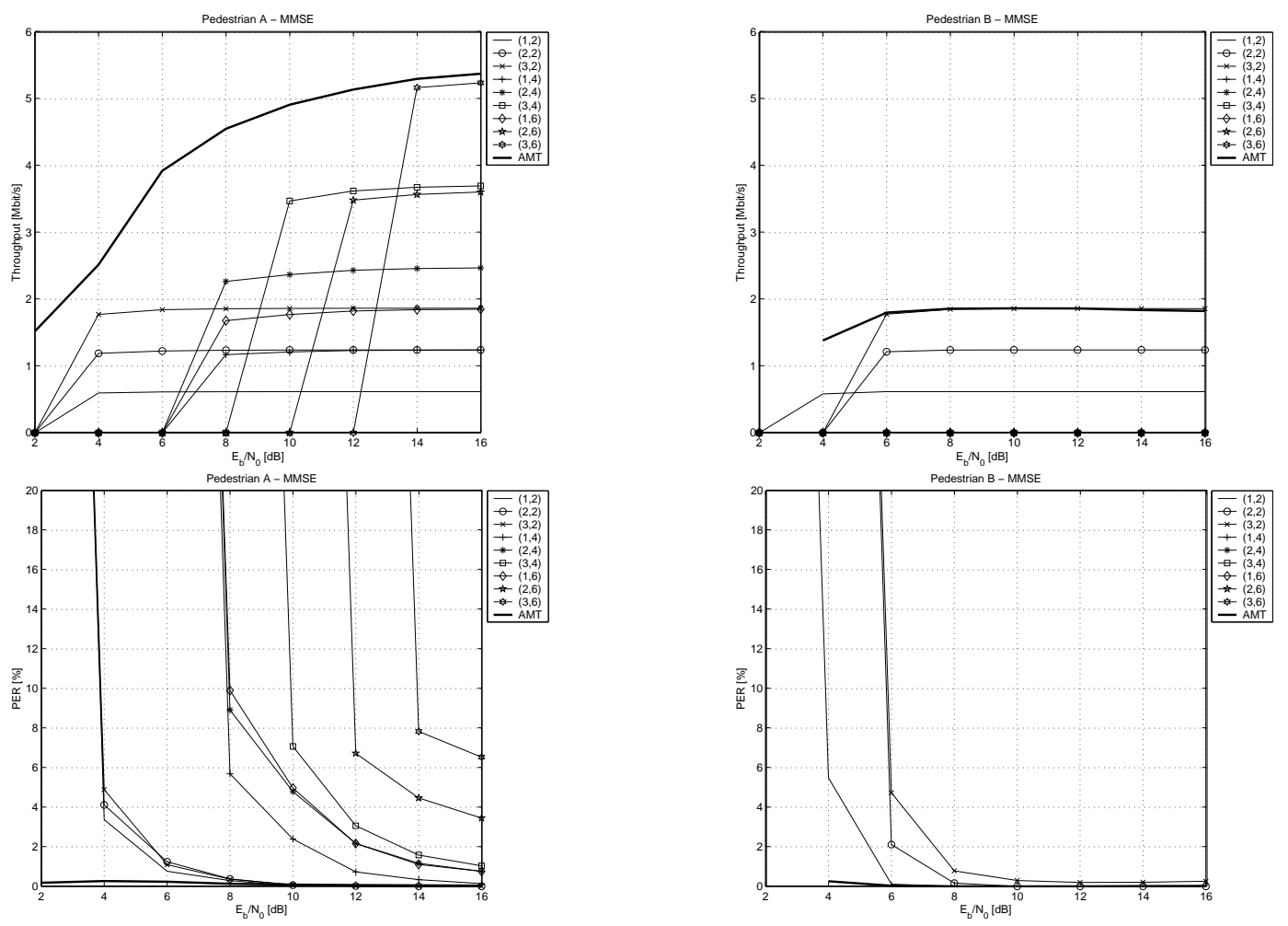

Figure 2: Simulation results (throughput and PER) for the multiuser MMSE receiver. 